\title{
Maria Cea D'Ancona, La activacion de la xenofobia: ¿que miden las encuestas?
}

\section{J.J. Igartua Perosanz}

\section{(2) OpenEdition}

\section{Journals}

Édition électronique

URL : http://journals.openedition.org/travailemploi/4327

DOI : $10.4000 /$ travailemploi.4327

ISSN : 1775-416X

Éditeur

DARES - Ministère du Travail

\section{Édition imprimée}

Date de publication : 15 septembre 2008

Pagination : 122-123

ISSN : 0224-4365

\section{Référence électronique}

J.J. Igartua Perosanz, « Maria Cea D'Ancona, La activacion de la xenofobia: ¿que miden las encuestas?», Travail et Emploi [En ligne], 115 | juillet-septembre 2008, mis en ligne le 18 février 2011, consulté le 22 septembre 2020. URL : http://journals.openedition.org/travailemploi/4327 ; DOI : https://doi.org/ 10.4000/travailemploi.4327 


\section{La activacion de la xenofobia en Espana \& Que miden las encuestas?}

\author{
(L'activation de la xénophobie en \\ Espagne. Que mesurent les enquêtes?) \\ Maria Cea D'Ancona M. A.
}

\author{
Madrid, CIS-Siglo XXI, Colección \\ «Monografías 》 (collection \\ «Monographies»), no 210, 2004
}

\section{Lu par J.J. Igartua Perosanz (université de Salamanque)}

Le titre choisi par Maria Ángeles Cea D’Ancona (professeur de sociologie à l'université Complutense de Madrid) pour son texte retient l'attention: L'activation de la xénophobie en Espagne. Peutêtre le mot «xénophobie» qui littéralement signifie «haine, répugnance ou hostilité envers les étrangers » (selon le dictionnaire de la langue espagnole de l'Académie royale espagnole (Real Academia)), est-il un terme trop dur pour se situer dans l'optique d'un livre qui prétend radiographier les attitudes des Espagnols vis-à-vis de l'immigration. Après tout, il renvoie à un trait négatif que peu de personnes revendiquent. Cependant, le terme xénophobie s'accompagne d'un autre (l'activation) et ils forment ensemble un tandem dont l'intérêt est incontournable pour le sociologue: comment germent, grandissent et évoluent les attitudes envers les immigrants.

Le contenu du texte de Maria Angeles Cea D’Ancona résume l'état des connaissances de la recherche en sociologie sur le racisme en Espagne et dans l'Union européenne. Une étude rigoureuse sur la mesure du racisme y est présentée ainsi que sur les techniques d'analyse les plus efficaces dédiées à l'étude d'un concept complexe et multivarié: la xénophobie. Pour ce faire, elle place son "cadre théorique», à partir duquel un modèle de mesure et une opérationalisation des variables sont déduits, au sein des études sur les préjugés subtils et les préjugés manifestes, de Pettigrew et Meertens, auteurs dont le travail de 1995, publié dans la revue European Journal of Social Psychology, est devenu une référence incontournable pour tous les chercheurs en quête d'analyse du phénomène que constitue le racisme. Le livre de Cea D'Ancona se compose d'une introduction, de trois chapitres, sans oublier les conclusions et un appendice (qui donne des résultats incorporant les informations des enquêtes les plus récentes des années 2003 et 2004). Le texte se présente sous la forme d'un rapport d'enquête et, du point de vue méthodologique, il s'appuie de manière très claire sur l'enquête et l'analyse de données quantitatives, tout en présentant des résultats d'études qualitatives, plus particulièrement en ayant recours à la technique des groupes de discussion.
L'introduction campe les objectifs du travail de recherche, à savoir l'analyse de l'opinion publique face à l'immigration, en prenant comme référence les enquêtes spécifiques sur l'immigration et les baromètres mensuels du Centre d'enquêtes sociologiques. Toutefois, d'autres enquêtes nationales et européennes sont mobilisées, y compris celles émanant de la Commission européenne (eurobaromètres), ce qui permet de valider les résultats dans bien des cas et dans d'autres, de passer à une phase de critique méthodologique sur la formulation des énoncés des questions et des catégories de réponses ou sur la désirabilité sociale, l'un des principaux problèmes qui se posent dès lors qu'il s'agit de mesurer le racisme. Même si cela ne constitue pas un objet spécifique de son analyse, Cea D’Ancona se penche également (à certaines occasions en fondant son jugement sur des études d'analyses de contenu, très rares en Espagne) sur le rôle que jouent les moyens de communication de masse dans l'«activation » de la xénophobie. De fait, à la seconde page de l'introduction, l'auteur fait remarquer que l'identification de l'immigration comme «problème» ressort fréquemment dans l'opinion publique et que «l'augmentation de rapports à caractère négatif ou ambivalent sur l'immigration dans les moyens de communication sociale contribue à ce phénomène» p. ?? ?X). Dans ce cadre, la capacité d'influence des moyens de communication permet par là même à l'immigration de devenir un problème, tout en gommant sa vraie nature polyédrique, qu'il s'agisse là d'un processus ou d'un phénomène social qui contribue à la richesse sociale, culturelle et économique.

Le premier chapitre (enquêtes sur les attitudes face à l'immigration en Espagne et en Europe) contextualise l'étude du racisme dans l'environnement européen d'un point de vue historique en signalant les principaux jalons de la recherche sur ce thème au niveau européen et, de manière plus spécifique, en Espagne. Dans le second chapitre (typologie des attitudes: entre tolérance, ambivalence et refus), Cea D'Ancona introduit une première analyse des attitudes envers l'immigration, en prenant comme référence les données de l'Eurobaromètre 53 pour l'année 2000, ce qui permet d'obtenir une première classification des pays qui composaient alors l'Union Européenne (quinze au total) et d'aboutir à une première conclusion: l'Espagne "se situe au deuxième rang des pays les plus tolérants dans l'Union européenne» (p. 14). Cependant, l'auteur considère que l'Eurobaromètre 53 pâtit de graves déficiences méthodologiques autorisant à mettre en doute la validité de ces résultats. D’où la nécessité, pour savoir si les Espagnols sont aussi tolérants qu'il y paraît face à l'immigration, comme l'Eurobaromètre 53 semble le dire, de lancer une nouvelle analyse à partir des données les plus récentes recueillies par le CES dans l'enquête spécifique de 1996 sur ce thème et au niveau des eurobaromètres qui incluaient des questions pertinentes sur l'immigration et le racisme (2000, 2001 et 2002). Ainsi cherche-t-on à vérifier si au cours d'une période 
marquée par la croissance de la population étrangère en Espagne, les attitudes envers les immigrants ont connu des changements. Un premier résultat qui ressort de ces analyses est l'augmentation de la proportion de personnes considérées comme étant récalcitrantes à l'immigration (de 8\% en juin 1996 à $28 \%$ en juin 2002) et, par voie de conséquence, le développement des attitudes de refus envers la population étrangère. Il ressort également de tout cela que le CES (et plus spécialement, le baromètre de février 2000) a permis, mieux que l'Eurobaromètre 53 (réalisé en avril-mai 2000), de capter les attitudes d'intolérance vis-à-vis des immigrants.

Le troisième chapitre (évolution de la xénophobie: analyse détaillée de ses dimensions à partir d'enquête) constitue la partie la plus volumineuse: elle permet de passer en revue les principales dimensions de la xénophobie. Plus particulièrement, les différentes dimensions latentes explicatives de la xénophobie en Espagne sont passées en revue, dimensions captées grâce à l'application de techniques d'analyse variées (analyse factorielle en composantes principales). Dix facteurs latents peuvent être identifiés au total comme indicateurs de racisme disposant chacun d'une notion qui lui est propre (la méthode de rotation orthogonale varimax étant appliquée): attitudes vis-à-vis des droits sociaux des immigrants, attitudes vis-à-vis des droits liés à la citoyenneté, niveau de sociabilité face aux immigrants, attitudes face à la politique d'immigration, clichés négatifs sur l'immigration, discrimination ethnique, prise de position face aux partis racistes, acceptation de l'immigré, violence faite aux immigrants et peur de la sédentarisation des immigrés. Ce troisième chapitre fournit une analyse plus détaillée de la xénophobie et des facteurs pouvant l'expliquer. Pour ce faire, l'auteur a recours à l'analyse de variance afin de vérifier dans quelle mesure les attitudes de refus ou d'acceptation de l'immigré sont modulées selon le sexe, l'âge, le niveau d'études, l'emploi, la situation professionnelle, le type de travail, la catégorie sociale, les revenus, l’idéologie politique, la religiosité ou encore la taille du logement, mais aussi la densité de la population immigrée au lieu de résidence des personnes interrogées (communauté autonome de résidence).

Dans ses conclusions, Cea D'Ancona résume les principaux résultats obtenus en procédant à une analyse des différentes enquêtes portant sur les attitudes face à l'immigration en Espagne. Il ressort qu'en Espagne, le racisme croît en même temps que le choix de l'Espagne comme un pays de destination pour les immigrés économiques. On observe également l'existence de divergences significatives en fonction de certaines variables sociodémographiques: «Refus de l'immigration plus manifeste chez les personnes plus âgées (plus spécifiquement, les retraités), les personnes au faible niveau d'études, celles idéologiquement tournées vers la droite, les catholiques pratiquants et les personnes de moindre statut social» (p. 288). Le poids explicatif de chacune de ces variables, en prenant comme indi- cateur le pourcentage de variance expliquée $\left(R^{2}\right)$ se situe entre $2 \%$ et $4 \%$ dans la majorité des cas (même si la variable niveau d'études explique jusqu'à $9 \%$ de la variance pour certaines dimensions). D’autre part, on constate également que tous les immigrés ne sont pas considérés à l'identique, les immigrés originaires du Maroc subissant le plus fort rejet et ceux d'Amérique latine étant mieux considérés. Des différences dans les attitudes face à l'immigration sont encore observées parmi les personnes interrogées résidant dans des régions à forte ou à faible densité de population immigrée: «La différence résultant du fait de vivre dans telle ou telle communauté autonome se vérifie là. Dans les communautés ayant connu une augmentation plus forte de la population immigrée dans les dernières années, surtout marocaine, le refus face à l'immigration est plus manifeste [...]. Il semble que l'expérience de cohabitation avec des minorités ethniques et culturelles différentes n'implique pas nécessairement une plus grande confraternité entre les différentes ethnies et cultures. Au contraire, cela réveille des sentiments xénophobes favorisés par des préjugés ethniques» (p. 288).

L'auteur conclut en affirmant qu'il sera nécessaire de continuer à mener des études empiriques sur les facteurs qui favorisent ou activent la xénophobie. De ce point de vue, à plus d'un endroit du livre, des hypothèses intéressantes sont présentées, congruentes de surcroît avec le développement disciplinaire de la communication, sur la contribution possible des moyens de communication (en particulier les moyens d'information) à l'activation de la xénophobie. Cet état de fait converge avec des perspectives aussi diverses que celles de l'analyse critique du discours (Teun van Dijk étant une référence incontournable) ou ce qui ressort des perspectives d'Agenda Setting et de la théorie de la conceptualisation (Framing Theory). Ces deux dernières approches théoriques fondent leur stratégie méthodologique sur l'analyse de contenu (pour examiner la couverture et le traitement réservé à l'immigration) sur des enquêtes (afin d'analyser le lien entre consommation médiatique et perception des problèmes sociaux) ainsi que sur des recherches expérimentales qui permettent d'aller au-delà de la simple description des phénomènes, en analysant l'impact sociocognitif des théories de conceptualisation de l'immigration (Framing Theories) en même temps que sont analysés les processus et les mécanismes expliquant l'influence des médias.

En résumé, le lecteur trouvera entre ses mains un texte excellent et rigoureux sur la mesure du racisme, intégrant quantité de données sur son développement accéléré (sans nuances) dans la société espagnole et sur ses possibles déterminants, et qui constitue un ouvrage incontournable pour tous ceux qui travaillent sur un sujet (le racisme) qui de nouveau, et plus d'un demi-siècle après la publication de The nature of prejudice de Gordon W. Allport, resurgit dans l'actualité des agendas scientifiques. 